\title{
Desafios à ampliação da cobertura dafluoretação da água em municípios brasileiros com mais de 50 mil habitantes na primeira metade do século XXI
}

\section{Challenges to spreading water fluoridation} coverage in Brazilian municipalities with 50 thousand inhabitants or more at first half of XXI century

\section{Desafíos para el aumento de la cobertura de la fluoruración del agua en municipios brasileños con más de 50 mil habitantes en la primera mitad del siglo XXI}

Angelo Giuseppe Roncalli Luiz Roberto Augusto Noro ${ }^{1}$

Celso Zilbovicius ${ }^{2}$

Helenita Corrêa Ely ${ }^{3}$ Helder Henrique Costa Pinheiro ${ }^{4}$

Paulo Capel Narvai ${ }^{2}$

Paulo Frazão ${ }^{2}$

RESUMO: Embora a fluoretação da água de abastecimento público seja um importante determinante para explicar o declínio da cárie dentária nos países que implementaram sua provisão, é grande o desafio para assegurar sua expansão para todas as regiões e territórios. O objetivo do estudo foi determinar as características dos municípios brasileiros com mais de 50 mil habitantes correlacionadas com a cobertura da fluoretação da água. Estudo ecológico transversal com 614 municípios cujo desfecho é representado pela cobertura populacional da política pública e as variáveis independentes referem-se a indicadores municipais socioeconômicos, de saneamento e

\footnotetext{
1 Universidade Federal do Rio Grande do Norte

2 Universidade de São Paulo

3 Pontifícia Universidade Católica do Rio Grande do Sul

4 Universidade Federal do Pará
} 
de serviços de atenção primária à saúde. As relações foram analisadas por meio de regressão linear múltipla. $\mathrm{Na}$ análise ajustada $\left(\mathrm{R}^{2}=0,39\right)$, a cobertura populacional correlacionou-se positivamente com a renda familiar percapita e negativamente com desigualdade da renda, água e esgoto inadequados, cobertura potencial da saúde bucal na estratégia saúde da família. Nas regiões Sudeste, Sul e Centro-Oeste $\left(\mathrm{R}^{2}=0,33\right)$, analfabetismo e nível de desenvolvimento humano mostraram correlação positiva, enquanto desigualdade de renda e saneamento inadequado apresentaram correlação negativa. Já nas regiões Norte e Nordeste $\left(\mathrm{R}^{2}=0,40\right)$, saneamento inadequado e nível de desenvolvimento humano mostraram correlação negativa. As desigualdades na expansão dos serviços de saneamento e dos sistemas de vigilância da concentração do fluoreto entre as regiões brasileiras trazem importantes desafios nas próximas décadas que vão requerer sua inclusão na agenda regulatória dos respectivos setores e a implementação de mecanismos de coordenação intersetorial da política pública.

Palavras-Chave: Política Pública, Saúde Bucal, Fluoretação.

\begin{abstract}
Although fluoridation of public water is an important determinant to explain the decline in dental caries in countries that have implemented it, the challenge to ensure its expansion to all regions and territories is great. The objective of this study was to determine the characteristics of Brazilian municipalities with more than 50,000 inhabitants correlated with water fluoridation coverage. Method: Cross-sectional ecological study encompassing Brazilian municipalities with more than 50,000 inhabitants in which the outcome is represented by population coverage of the public policy and the independent variables refer to municipal socioeconomic, sanitation and primary health care services indicators. Relations were analyzed by multiple linear regression. Results: 614 municipalities were analyzed. In the adjusted analysis $\left(\mathrm{R}^{2}=0.39\right)$, population coverage correlated positively with per capita household income and negatively correlated with income inequality, inadequate water and sewage, potential oral health coverage in the family health strategy. While illiteracy and human development level showed positive correlation and income inequality and inadequate sanitation, negative correlation in the Southeast, South and Central West $\left(\mathrm{R}^{2}=0.33\right)$ Brazilian regions; in the North and Northeast $\left(\mathrm{R}^{2}=0.40\right)$ regions, inadequate sanitation and human development level showed a negative correlation. Inequalities in the expansion of sanitation services and fluoride concentration adjustment systems among Brazilian regions pose important challenges in the coming decades that will require their inclusion in the regulatory agenda of the corresponding sectors and the implementation of intersectoral coordination mechanisms for public policy.
\end{abstract}

Keywords: Public Policy, Oral Health, Water Fluoridation

RESUMEN: Aunque la fluoruración del agua proveniente del abastecimiento público sea un determinante importante del declive de la prevalencia de caries en los países que han implementado su provisión, es grande el desafío para garantizar su expansión a todas las regiones y territorios. El objetivo de este estudio fue determinar las características de los municipios brasileños con 
más de 50.000 habitantes en relación con la cobertura de fluoruración del agua. Estudio ecológico transversal que abarcó 614 municipios. La variable dependiente es la población cubierta por la política pública y las variables independientes se refieren a los indicadores municipales socioeconómicos, de saneamiento y de servicios de atención primaria de salud. Las relaciones se analizaron utilizando una regresión lineal múltiple. En el análisis ajustado $\left(\mathrm{R}^{2}=0,39\right)$, la cobertura de la población mostró una correlación positiva con el ingreso familiar per cápita y negativa con la insuficiencia de ingresos, la desigualdad de agua y alcantarillado, y la cobertura potencial de salud bucal en la estrategia de salud familiar. En las regiones Sureste, Sur y Centro Oeste $\left(\mathrm{R}^{2}=0,33\right)$, el analfabetismo y el nivel de desarrollo humano mostraron una correlación positiva, mientras que la desigualdad de ingresos y el saneamiento inadecuado presentaron una correlación negativa. Por otro lado, las regiones Norte y Noreste $\left(\mathrm{R}^{2}=0,40\right)$ mostraron una correlación negativa entre el saneamiento inadecuado y el nivel de desarrollo humano. Las desigualdades tanto en la expansión de los servicios de saneamiento cuanto en los sistemas de vigilancia de concentración de fluoruro entre las regiones brasileñas plantean retos importantes en las próximas décadas que requerirán su inclusión en la agenda reguladora de los sectores respectivos, así como la implementación de mecanismos de coordinación intersectorial de esa política pública.

Palabras clave: Política Pública. Salud Oral. Fluoruración.

\section{INTRODUÇÃO}

Tecnologia de saúde pública cuja eficácia, efetividade e segurança tem sido atestada por investigações realizadas durante mais de $80 \operatorname{anos}^{1,2}$, a fluoretação da água de abastecimento público é uma das principais linhas de ação da Política Nacional de Saúde Bucal (PNSB) brasileira desde o início dos anos $1950^{3,4}$, constituindono início do século XXI um dos seus pilares estratégicos, tendo em vista sua contribuição para diminuir desigualdades e promover a equidade em saúde bucal ${ }^{5}$. Ademais, é reconhecido o seu potencial para, ainda que operando preventivamente em relação à cárie dentária, induzir mudanças qualitativas tanto no tratamento da água para consumo humano, quanto nas medidas de sua vigilância sanitária ${ }^{6}$.

Não obstante, essa tecnologia,de modo análogo às outras medidas de saúde pública, vem sendo ameaçada pela disseminação da desinformação na internet e nas redes sociais ${ }^{7,8}$. No Brasil,não são raras as iniciativas noCongresso Nacionalpara revogar a lei 6.050/19749. Uma solução de continuidade da bem-sucedida política pública de fluoretação das águas no Brasil, decorrente de decisões legislativas desinformadas, poderiam ter consequências prejudiciais à saúde bucal da população ${ }^{10}$.

Pesquisas brasileiras do início do século XXI indicam a necessidade de redobrar os esforços para ampliar a fluoretação das águas nos municípios das regiões Norte e Nordeste, com vistas a diminuir as desigualdades observadas no contexto nacional quanto ao acesso a essa medida ${ }^{11}$. A notável assimetria dessas regiões em relação ao Sul, Sudeste e Centro-Oeste chama a atenção e 
requer iniciativas políticas tendentes a diminuir tais desigualdades, atenuando-as, pelo menos, numa primeira etapa ${ }^{12}$.

Além dessas diferenças no plano macrorregional, estudos têm mostradoiniquidadesna provisão da política pública entre os municípios conforme o porte demográfico, o nível de desenvolvimento humano, a cobertura da rede de água e esgoto e o tipo de companhia de saneamento ${ }^{13,14,15}$. Entretanto, nenhum estudo investigou a relação entre indicadores municipais e a porcentagem da população coberta pelo benefício.

Assim, o objetivo do presente estudo foi determinar as características dos municípios brasileiros com mais de 50 mil habitantes correlacionadas com a cobertura da fluoretação da água em sistema de abastecimento público.

\section{MÉTODO}

Trata-se de um estudo ecológico que tomou como unidade de análise todos os municípios brasileiros com mais de 50 mil habitantes no ano de 2012 segundo asprojeções intercensitárias (1981 a 2012) disponibilizadas pelo Departamento de Informática do Sistema Único de Saúde (DATASUS) a partir das estimativas populacionais do Instituto Brasileiro de Geografia e Estatística (IBGE). Estas unidades de observação fizeram parte da pesquisa "Cobertura e Vigilância da Fluoretação da Água no Brasil" também conhecido como "Projeto VIGIFLUOR"16. No contexto brasileiro, os municípios com mais de 50 mil habitantes, ocupam papel central na agenda política nacional, seja no plano econômico e cultural, seja no âmbito institucional do sistema de saúde, uma vez que emergem como importantes polos regionais, em torno dos quais o Sistema Único de Saúde (SUS) organiza ações e serviços sanitários dando forma às regiões de saúde.

\section{Fonte dos dados}

A fonte principal dos dados tem como origem o banco de dados do Projeto VIGIFLUOR, onde os dados de cobertura da fluoretação foram obtidos para os municípios brasileiros com mais de 50 mil habitantes. Os critérios para o cálculo da cobertura estão descritos em outra publicação ${ }^{16}$ e tiveram por base o IBGE e o Sistema de Informação de Vigilância da Qualidade da Água de Consumo Humano (SISAGUA).A este banco de dados foram acrescidos, por intermédio de linkagedeterminístico, variáveis socioeconômicas oriundas do Atlas de Desenvolvimento Humano no Brasil, o qual contém indicadores calculados a partir do censo 2010. Outras variáveis relativas aos serviços de saúde foram obtidas após consulta ao sítio do DATASUS, no módulo e-Gestor da Atenção Básica (https://egestorab.saude.gov.br/).

\section{Processamento e análise dos dados}

O desfecho foi definido pela porcentagem de população coberta pela fluoretação em sistema público de abastecimento de água. As variáveis independentes referem-se a indicadores Tempus, actas de saúde colet, Brasília, 14(1), 161-173 mar, 2020. Epub Mai/2020 ISSN 1982-8829 
municipais socioeconômicos, de saneamento, e de serviços de atenção primária à saúde. Após o linkage determinístico dos bancos de dados, as variáveis foram ajustadas para a análise a partir de sua recodificação em tercis, quando necessário. Inicialmente, como procedimento prévio para a regressão linear múltipla, foi realizada uma análise de correlação a partir do cálculo do coeficiente de correlação de Pearson e respectiva significância para todas as variáveis de interesse. Com a obtenção da matriz de correlação, foi possível selecionar as principais variáveis correlacionadas com a variável dependente, a partir da exclusão daquelas que não foram significativas ou que obtiveram um valor muito baixo de correlação. Além disso, foram excluídas as variáveis independentes que apresentaram alta colinearidade entre si e, para alguns casos, foi escolhida a variável com maior valor de correlação com a variável dependente. O conjunto final de variáveis incluídas na presente análise está descrito no Quadro 1.

Em seguida foi realizada uma análise descritiva, por intermédio do cálculo das médias e intervalos de confiança da variável "cobertura de fluoretação" em relação às demais variáveis independentes categorizadas em tercis.

Quadro 1. Variáveis utilizadas no estudo e respectivas fontes.

\begin{tabular}{|c|c|c|c|}
\hline Variável & Descrição & Origem & Escala de medida \\
\hline \multicolumn{4}{|l|}{ Variável Dependente } \\
\hline $\begin{array}{l}\text { Cobertura de } \\
\text { fluoretação das águas }\end{array}$ & $\begin{array}{l}\text { Percentual de cobertura populacional } \\
\text { de água fluoretada no município no } \\
\text { ano de } 2014\end{array}$ & $\begin{array}{l}\text { Projeto } \\
\text { VIGIFLUOR }\end{array}$ & $\%(0$ a 100$)$ \\
\hline \multicolumn{4}{|c|}{ Variáveis independentes socioeconômicas } \\
\hline Região natural & $\begin{array}{l}\text { Região brasileira segundo } \\
\text { classificação do IBGE a partir de } \\
\text { características geográficas }\end{array}$ & $\begin{array}{l}\text { Projeto } \\
\text { VIGIFLUOR }\end{array}$ & $\begin{array}{l}\text { Norte, Nordeste, Sudeste, } \\
\text { Sul e Centro-Oeste, } \\
\text { reagrupadas para } 0=\text { Sul, } \\
\text { Sudeste e Centro-Oeste e } \\
1=\text { Norte e Nordeste }\end{array}$ \\
\hline Analfabetismo & $\begin{array}{l}\text { Percentual da população analfabeta } \\
\text { com } 15 \text { anos ou mais de idade }\end{array}$ & $\begin{array}{l}\text { IBGE/Atlas de } \\
\text { Desenvolvimento } \\
\text { Humano }\end{array}$ & $\%(0$ a 100$)$ \\
\hline Índice de Gini & $\begin{array}{l}\text { Indicador de concentração de renda, } \\
\text { ilustrando desde uma distribuição } \\
\text { inteiramente igualitária (0) a uma } \\
\text { concentração de renda em um único } \\
\text { indivíduo (1) }\end{array}$ & $\begin{array}{l}\text { IBGE/Atlas de } \\
\text { Desenvolvimento } \\
\text { Humano }\end{array}$ & 0 a 1 \\
\hline Renda per capita & $\begin{array}{l}\text { Média da renda familiar mensal } \\
\text { em reais dividida pelo número de } \\
\text { moradores }\end{array}$ & $\begin{array}{l}\text { IBGE/Atlas de } \\
\text { Desenvolvimento } \\
\text { Humano }\end{array}$ & Reais $(\mathrm{R} \$)$ \\
\hline Saneamento inadequado & $\begin{array}{l}\text { Percentual de domicílios com } \\
\text { abastecimento de água e esgoto } \\
\text { inadequados em um dado município }\end{array}$ & $\begin{array}{l}\text { IBGE/Atlas de } \\
\text { Desenvolvimento } \\
\text { Humano }\end{array}$ & $\%(0$ a 100$)$ \\
\hline $\begin{array}{l}\text { Índice de } \\
\text { Desenvolvimento } \\
\text { Humano }\end{array}$ & $\begin{array}{l}\text { Indicador agregado, formado pelas } \\
\text { variáveis renda, longevidade e } \\
\text { educação }\end{array}$ & $\begin{array}{l}\text { IBGE/Atlas de } \\
\text { Desenvolvimento } \\
\text { Humano }\end{array}$ & 0 a 1 \\
\hline
\end{tabular}




\begin{tabular}{|l|l|l|l|}
\hline $\begin{array}{l}\text { Cobertura de SB na } \\
\text { Atenção Básica }\end{array}$ & $\begin{array}{l}\text { Percentual da população coberta por } \\
\text { equipes de saúde bucal na Atenção } \\
\text { Básica, vinculada ou não à ESF }\end{array}$ & $\begin{array}{l}\text { DATASUS/e- } \\
\text { Gestor }\end{array}$ & 0 a 100 \\
\hline Cobertura SB-ESF & $\begin{array}{l}\text { Percentual da população coberta por } \\
\text { equipes de saúde bucal na Estratégia } \\
\text { Saúde da Família }\end{array}$ & $\begin{array}{l}\text { DATASUS/e- } \\
\text { Gestor }\end{array}$ & 0 a 100 \\
\hline $\begin{array}{l}\text { Investimentos em } \\
\text { Atenção Básica }\end{array}$ & $\begin{array}{l}\text { Total investido em Atenção Básica } \\
\text { no ano de 2014 dividido pela } \\
\text { população do município }\end{array}$ & $\begin{array}{l}\text { DATASUS/e- } \\
\text { Gestor }\end{array}$ & Reais (R\$) per capita \\
\hline $\begin{array}{l}\text { Equipamentos } \\
\text { Odontológicos por } \\
\text { habitante }\end{array}$ & $\begin{array}{l}\text { Número de equipamentos } \\
\text { odontológicos do SUS por } 10 \text { mil } \\
\text { habitantes }\end{array}$ & CNES/IBGE & $\begin{array}{l}\text { Taxa por } 10 \text { mil } \\
\text { habitantes }\end{array}$ \\
\hline
\end{tabular}

Nota: IBGE=Instituto Brasileiro de Geografia e Estatística; SB=Saúde Bucal; ESF=Estratégia Saúde da Família; SUS=Sistema Único de Saúde; DATASUS=Departamento de Informática do SUS; CNES=Cadastro Nacional de Estabelecimentos de Saúde.

Para a obtenção do modelo de regressão linear múltipla, as variáveis foram incluídas pela técnica stepwise, sendo retiradas do modelo as variáveis com perda de significância até o limite de $p=0,10$, seguindo a sequência até a obtenção do modelo final. A variável "região" apresentou uma forte associação na análise bivariada, porém por se constituir em uma variável agregada, não poderia ser incluída no modelo de regressão. Desse modo, optou-se por uma análise estratificada, criando-se dois estratos:Norte/Nordestee Sudeste/Sul/Centro-Oeste a fim de verificar possíveis diferenças.

\section{RESULTADOS}

A população do estudo foi composta por 614 municípios. A cobertura média foi $58,2 \%$ e o valor da mediana foi $80,0 \%$. Do total, 168 (27,4\%) municípios não eram providos pela política pública. A Figura 1 ilustra as médias e respectivos intervalos de confiança (95\%) para a cobertura de fluoretação das águas em relação a algumas variáveis selecionadas. Observa-se uma diferença marcante entre as regiões e uma relação direta com indicadores socioeconômicos. Para as variáveis de serviços de saúde (investimentos em Atenção Básica e cobertura de saúde bucal na ESF) observa-se o inverso, ou seja, os maiores percentuais de cobertura se encontram em municípios com menores investimentos em atenção básica e com menores taxas de cobertura de saúde bucal na Estratégia Saúde da Família. 
Figura1. Médias e respectivos intervalos de confiança (95\%) da cobertura populacional de fluoretação das águas segundo variáveis selecionadascategorizadas em tercis. Fonte: Elaboração dos autores.
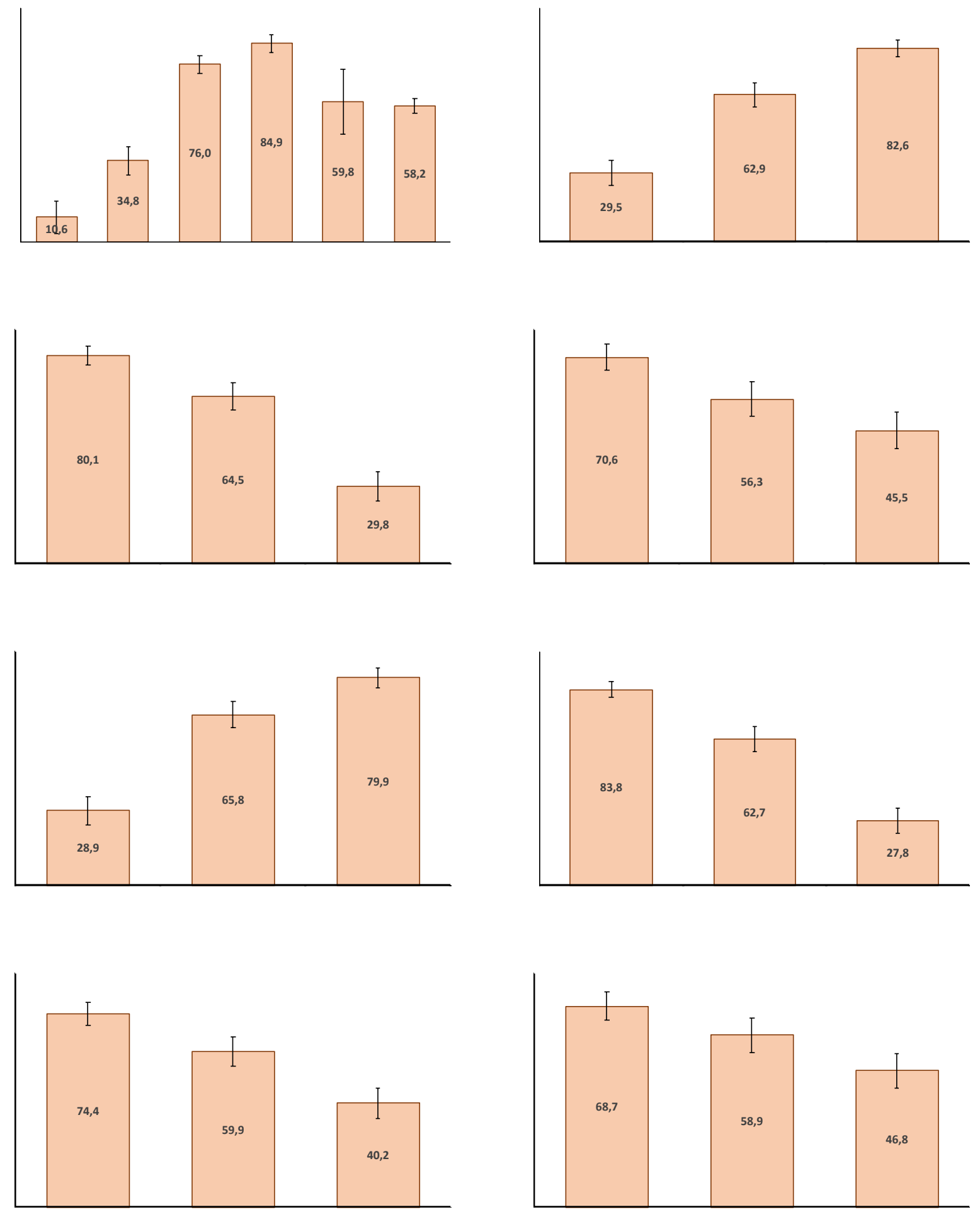

Na Tabela 1, observam-se três modelos de regressão, um geral, um para as regiões Norte e Nordeste e um para as regiões Sudeste, Sul e Centro-Oeste. No modelo 1 para todas as regiões, 
renda per capita apresenta correlação positiva enquanto desigualdade de renda (índice de Gini), saneamento inadequado e cobertura potencial da saúde bucal na Estratégia Saúde da Família mostraram correlação positiva com o desfecho. Nível de desenvolvimento humano e de investimento na atenção básica e analfabetismo perderam significância no modelo final.

No modelo 2 para as regiões Sudeste, Sul e Centro-Oeste, mantiveram-se no modelo final as variáveis "analfabetismo", "índice de Gini", "saneamento inadequado" e "IDH". Para o modelo 3 , relativo às regiões Norte e Nordeste, o resultado final contou somente com as variáveis "água e esgoto inadequados" e "IDH". É interessante notar, entretanto, que no modelo 2, o coeficiente de regressão para o IDH foi positivo e no modelo 3 (para as regiões Norte e Nordeste) foi negativo. Enquanto no Norte/Nordeste, para cada 0,1 ponto no IDH, observou-se uma redução de 1,5\%, no Sudeste/Sul/Centro-Oeste observou-se um aumento de 3,0\% na porcentagem de cobertura. Saneamento inadequado foi a única variável correlacionada negativamente nos três modelos. Enquanto no modelo geral, para cada unidade de aumento na porcentagem de domicílios com abastecimento de água e esgoto inadequados, a porcentagem de redução da cobertura foi 1,1\%, no Norte/Nordeste foi 1,4\% e no SudesteSul/Centro-Oeste, a redução foi 4,1\%.

Tabela 1. Resultados da Regressão Linear Múltipla para a cobertura de fluoretação das águas em municípios com mais de 50 mil habitantes estratificada por região.

\begin{tabular}{|c|c|c|c|c|c|c|}
\hline Variável & Coeficiente & IC $(95 \%)$ & $\mathrm{p}$ & Coeficiente & IC $(95 \%)$ & $\mathrm{p}$ \\
\hline Modelo para todas as regiões & \multicolumn{3}{|c|}{ Modelo inicial não ajustado } & \multicolumn{3}{|c|}{ Modelo final ajustado $\left(\mathrm{R}^{2}=0,388\right)$} \\
\hline Constante & & & & 91,85 & 66,$48 ; 117,22$ & $<0,001$ \\
\hline Analfabetismo & $-2,40$ & $-2,77 ;-2,03$ & $<0,001$ & - & - & - \\
\hline Índice de Gini & $-202,49$ & $-256,86 ;-148,13$ & $<0,001$ & $-86,49$ & $-139,36 ;-33,63$ & 0,001 \\
\hline Renda percapita & 0,06 & 0,$05 ; 0,07$ & $<0,001$ & 0,03 & 0,$02 ; 0,04$ & $<0,001$ \\
\hline Água e esgoto inadequados & $-1,91$ & $-2,15 ;-1,67$ & $<0,001$ & $-1,14$ & $-1,47 ;-0,82$ & $<0,001$ \\
\hline IDH & 315,30 & 275,$36 ; 355,24$ & $<0,001$ & - & - & - \\
\hline Cobertura SB-ESF & $-0,37$ & $-0,48 ;-0,26$ & $<0,001$ & $-0,13$ & $-0,23 ;-0,02$ & 0,017 \\
\hline Investimento em AB percapita & $-1,35$ & $-1,64 ;-1,06$ & $<0,001$ & - & - & - \\
\hline $\begin{array}{l}\text { Modelo para regiões Sudeste, Sul e } \\
\text { Centro-Oeste }(n=377)\end{array}$ & \multicolumn{3}{|c|}{ Modelo inicial não ajustado } & \multicolumn{3}{|c|}{ Modelo final ajustado $\left(\mathrm{R}^{2}=0,333\right)$} \\
\hline Constante & & & & $-125,05$ & $-219,42 ;-30,68$ & 0,010 \\
\hline Analfabetismo & 2,39 & 0,$12 ; 4,66$ & 0,039 & 2,63 & 0,$51 ; 4,75$ & 0,015 \\
\hline Índice de Gini & $-66,31$ & $-139,02 ; 6,40$ & 0,074 & $-68,80$ & $-133,32 ;-4,28$ & 0,037 \\
\hline Renda per capita & $-0,002$ & $-0,026 ; 0,021$ & 0,841 & - & - & - \\
\hline Água e esgoto inadequados & $-4,05$ & $-6,16 ;-1,93$ & $<0,001$ & $-4,12$ & $-6,22 ;-2,03$ & $<0,001$ \\
\hline IDH & 312,09 & 140,$17 ; 484,00$ & $<0,001$ & 300,97 & 170,$03 ; 431,86$ & $<0,001$ \\
\hline Cobertura SB-ESF & 0,029 & $-0,124 ; 0,182$ & 0,706 & - & - & - \\
\hline Investimento em $\mathrm{AB}$ per capita & 0,043 & $-0,398 ; 0,483$ & 0,848 & - & - & - \\
\hline $\begin{array}{l}\text { Modelo para as regiões Norte e } \\
\text { Nordeste }(n=237)\end{array}$ & \multicolumn{3}{|c|}{ Modelo inicial não ajustado } & \multicolumn{3}{|c|}{ Modelo final ajustado $\left(\mathrm{R}^{2}=0,402\right)$} \\
\hline Constante & & & & 146,99 & 74,$24 ; 219,74$ & $<0,001$ \\
\hline Analfabetismo & $-0,27$ & $-1,46 ; 0,918$ & 0,653 & - & - & - \\
\hline Índice de Gini & 105,22 & $-17,72 ; 228,16$ & 0,093 & - & - & - \\
\hline Renda per capita & $-0,006$ & $-0,067 ; 0,056$ & 0,858 & - & - & - \\
\hline Água e esgoto inadequados & $-1,63$ & $-2,18 ;-1,09$ & $<0,001$ & $-1,35$ & $-1,79 ;-0,92$ & $<0,001$ \\
\hline IDH & $-204,89$ & $-434,35 ; 24,58$ & 0,080 & $-149,21$ & $-253,50 ;-44,93$ & 0,005 \\
\hline
\end{tabular}




\section{DISCUSSÃO}

Investigou-se neste estudo, algumas características dos municípios com mais de 50 mil habitantes em relação àfluoretação da água. Os resultados mostraram que as características dos municípios expressas por indicadores socioeconômicos, de saneamento, e de serviços de atenção primária à saúdetêm importante poder preditivo $\left(\mathrm{R}^{2} \mathrm{aj}>0,30\right)$. De modo geral, a cobertura populacional desta medida foi maior em cidades cuja renda familiar percapitaera mais alta e a cobertura potencial da saúde bucal na estratégia saúde da família era mais baixa. Os indicadores de desigualdade social e econômica (Gini) e de saneamento inadequado (água e esgoto) se mostraram associados negativamente com a política pública.

Entretanto, na análise ajustada destacaram-se diferenças importantes entre as regiões brasileiras. O saneamento inadequado foi a única característica negativamente correlacionada com o desfecho nos três modelos investigados. A desigualdade de renda (índice de Gini) mostrou correlação negativa em dois modelos (geral e Sudeste/Sul/Centro-Oeste) e o índice de desenvolvimento humano mostrou correlação positiva no estrato das regiões Sudeste/Sul/Centro-Oeste e correlação negativa no Norte/Nordeste.

Este é o primeiro estudo que investigou a correlação deindicadores municipaissocioeconômicos, de saneamento, e de serviços de atenção primária à saúde, com a porcentagem de cobertura da fluoretação da água em municípios brasileiros com mais de 50 mil habitantes.Nos estudos anteriores, o desfecho foi a provisão da política pública e os resultados mostraram associação positiva com municípios pertencentes as regiões mais desenvolvidas,com elevado porte demográfico,alto e muito altonível de desenvolvimento humano e cobertura de água e esgoto acima do valor mediano ${ }^{12,13,14,15,17,18}$.

A principal contribuição foi mostrar que, quando se leva em consideração o contexto macrorregional brasileiro, as características dos municípios não têm o mesmo efeito em relação à porcentagem de população coberta pela fluoretação da água comparado à literatura científica sobre a provisão da política pública. Do ponto de vista desta literatura, era esperado que o analfabetismo, por ser maior em municípios de menor porte populacional e de baixo/muito baixo desenvolvimento humano, estaria negativamente correlacionado ao desfecho, e essa direção na relação entre as variáveis foi observada neste estudo na análise não ajustada contemplando todas as macrorregiões. Entretanto, quando se considera apenas as macrorregiões Sul/Sudeste/Centro-Oeste, observou-se uma correlação positiva modificada pela presença das variáveis relativas ao IDH, ao saneamento inadequado e à desigualdade de renda. Significa que neste estrato macrorregional, valores crescentes de cobertura foram encontrados em municípios com valores crescentes,tanto de analfabetismo acima de 15 anos de idade, como de desenvolvimento humano, e com valores decrescentes de desigualdade de renda e de saneamento inadequado.

ISSN 1982-8829 Tempus, actas de saúde colet, Brasília, 14(1), 161-173 mar, 2020. Epub Mai/2020 
Outro resultado inédito foi observado em relação aos valores do índice de desenvolvimento humano. Ao contrário da associação positiva entre provisão do benefício e nível de desenvolvimento humano, que se reproduziu no estrato macrorregional do Sul/Sudeste/Centro-Oestee na análise não ajustada contemplando todas as macrorregiões, observou-se nas macrorregiões Norte/ Nordeste uma correlação negativa ajustadapela variável relativa ao saneamento inadequado. Isso implica reconhecer que neste estrato macrorregional, na medida que aumentam os valores de coberturapopulacional diminuem os valores do índice de desenvolvimento humano e de saneamento inadequado.

A superação desse padrão assimétrico entre os estratos macrorregionais requer, na primeira metade do século XXI, o enfrentamento de vários desafios, dentre os quais a implementação de mecanismos de coordenação intersetorial dessa política pública na agenda regulatória dos respectivos setores. Para isto, porém, não bastará levar em consideração apenas a história da expansão do saneamento no país, cujos serviços alcançaram coberturas relevantes, primeiro nas regiões Sudeste e Sul, em comparação com as demais, o que conferiu uma trajetória de desigualdades territoriais ${ }^{19}$, mas também, aspectos específicos relativos ao processo de ampliação da cobertura da fluoretação da água. Fazê-lo é indispensável, uma vez, que tem importante relação de dependência, não apenas com indicadores municipais e com a região a que pertence o município ${ }^{20}$, mas também com o tipo de prestador do serviço de saneamento ${ }^{15}$.

Entre os alcances deste estudo, destaca-se o desenho ecológico que permite avaliar as características socioeconômicas e ambientais que afetama saúde e a implementação de políticas públicas em nível populacional. Por outro lado, o desenho não permite a produção de inferência em nível individual, e por utilizar diferentes bases de dados agregados está sujeito a problemas de acurácia e incompletude de dados. Para minimizar o efeito desses problemas, utilizou-se no presente estudo uma base de dados produzida pela triangulação de diferentes fontes de informação ${ }^{12}$.

Fatores contextuais relacionados ao perfil de desenvolvimento humano, à distribuição de renda e aos cuidados em saúde vêm sendo considerados essenciais para caracterizar a vulnerabilidade de grupos populacionais. As situações de iniquidade refletem falta de oportunidades e acesso a bens e serviços que atingem determinados grupos populacionais que vivem em certas localidades.Por conseguinte, as doenças e agravos na cavidade bucal também são determinados contextualmente pelos espaços geográficos e ambientais. Além da cobertura da fluoretação da água, o maior número de domicílios ligados a rede de abastecimento de água e IDH foram determinantes para prevalência mais baixa de cárie não tratada entre adolescentes da Região Sul e Sudeste, reconhecidas regiões como de maior desenvolvimento e riqueza ${ }^{21}$.

Cabe ressaltar que há declínio na cárie no Brasil, sendo atribuídoà expansão do acesso à águas fluoretadas como um dos fatores desta redução ${ }^{22}$,com acúmulo da doença em grupos com vulnerabilidades sociaise em regiões com menor desenvolvimento social, econômico e com aprofundamento das iniquidades. Políticas sociaisque melhoram o saneamento, como a ampliação 
do acesso à agua fluoretada, e a promoção demelhorias na educação, na renda e na longevidade estão relacionadas à melhoria dos níveis de saúde bucal em crianças brasileiras de $12 \operatorname{anos}^{23}$,denotandoa necessidade de investimentos para diminuição das desigualdades no referido acesso observada entre as regiões do país ${ }^{12}$.

Diante do exposto, conclui-se que indicadores municipais socioeconômicos, de saneamento, e de serviços de atenção primária à saúdetêm importante poder preditivo em relação à porcentagem de população coberta pela fluoretação da água.As desigualdades na expansão dos serviços de saneamento e dos sistemas de ajuste da concentração do fluoreto entre as regiões brasileiras trazem importantes desafios para as próximas décadas que vão requerer sua inclusão na agenda regulatória dos respectivos setores e a implementação de mecanismos de coordenação intersetorial da política pública.

\section{REFERÊNCIAS BIBLIOGRÁFICAS}

1) World Health Organization. Fluorine and fluorides. Geneva: WHO; 1984. (Environmental Health Criteria, 36).

2) Centers for Disease Control and Prevention. Achievements in public health, 1900-1999: Fluoridation of drinking water to prevent dental caries. MorbidityandMortalityWeeklyReports. 1999;48(41):933-40.

3) Freire PS. O problema da cárie dental no Brasil. Revista da Fundação SESP. 1970;15:89-97.

4) Narvai PC. Cárie dentária e flúor: uma relação do século XX. Ciência \& Saúde Coletiva. 2000;5(2):381-92.

5) Brasil. Ministério da Saúde. Secretaria de Atenção à Saúde. Departamento de Atenção Básica. Coordenação Nacional de Saúde Bucal. Diretrizes da Política Nacional de Saúde Bucal. Brasília: MS; 2004.

6) Frazão P, Ely HC, Noro LRA et al. O modelo de vigilância da água e a divulgação de indicadores de concentração de fluoreto. Saúdeem Debate. 2018;42(116):274-86.

7) Seymour, B., Getman, R., Saraf, A., Zhang, L. H., Kalenderian, E. (2015). When advocacy obscures accuracy online: digital pandemics of public health misinformation through an antifluoride case study. American JournalofPublic Health 105(3), 517-23.

8) Akerman M, Moysés SJ. Fluoretação da água de abastecimento e as políticas públicas de saúde. In: Frazão P, Narvai PC (orgs.). Cobertura e vigilância da fluoretação da água no Brasil: municípios com mais de 50 mil habitantes. São Paulo: Faculdade de Saúde da 
Universidade de São Paulo; 2017. p.5-32.

9) Zilbovicius C, Ferreira RGLA, Narvai PC. Água e saúde: fluoretação e revogação da Lei Federal no 6.050/1974. RevDirsanit. 2018;18(3)104-24.

10) Narvai PC, Frazão P, Fernandez RAC. Fluoretação da água e democracia. Saneas. 2004;2(18):29-33.

11) Narvai PC, Frazão P. Brasil: as marcas da desigualdade também na cobertura da fluoretação e na vigilância da água. In: Frazão P, Narvai PC (orgs.). Cobertura e vigilância da fluoretação da água no Brasil: municípios com mais de 50 mil habitantes. São Paulo: Faculdade de Saúde da Universidade de São Paulo; 2017. p.185-99.

12) Roncalli AG, Noro LRA, Cury JA, Zilbovicius C, Pinheiro HHC, Ely HC et al .Fluoretação da água no Brasil: distribuição regional e acurácia das informações sobre vigilância em municípios com mais de 50 mil habitantes. Cad. Saúde Pública. 2019; 35(6): $\mathrm{e} 00250118$.

13) Peres MA, Fernandes LS, Peres KG. Inequality of water fluoridation in Southern Brazil - the inverse equity hypothesis revisited. Social Science \& Medicine. 2004;58(6):1181-9.

14) Frazão $P$, Narvai PC. Water fluoridation in Brazilian cities at the first decade of the 21st century. Rev SaúdePública. 2017 May;51:47.

15) Silva FB, Frazão P. Sanitation utilities and fluoridation of water supply systems: an ecological study in Brazilian municipalities, 2008-2010. Epidemiologia e Serviços de Saúde. 2018; 27(4)e2018015.

16) Frazão P, Narvai PC (Orgs). Cobertura e vigilância da fluoretação da água no Brasil: municípios com mais de 50 mil habitantes. São Paulo: Faculdade de Saúde Pública da USP, 2017. 202p.

17) Antunes JLF, Narvai PC. Políticas de saúde bucal no Brasil e seu impacto sobre as desigualdades em saúde. Revista de Saúde Pública. 2010; 44: 360-365.

18) Gabardo MCL, Silva WJ, Olandoski M, Moysés ST, Moysés SJ. Inequalities in public water supply Fluoridation in Brazil: an ecological study. BMC Oral Health2008;8(9):1-7.

19) Arretche M. (Org.) Trajetória das desigualdades: como o Brasil mudou nos últimos cinquenta anos. São Paulo: Ed Unesp; CEM, 2015. 
20) Narvai PC, Frazão P. Epidemiologia, política, e saúde bucal coletiva. In: Antunes JLF, Peres MA. Epidemiologia da saúde bucal. Rio de Janeiro: Guanabara Koogan; 2006. p. 346-62.

21) Frias AC, Antunes JLF, Junqueira SR, Narvai PC. Determinantes individuais e contextuais da prevalência de cárie dentária não tratada no Brasil. Rev. Panam Salud Publica, v. 22, n. 4, p. 279-85, 2007.

22) Narvai PC, Frazão P, Roncalli AG, Antunes JLF. Cárie dentária no Brasil: declínio, polarização, iniquidade e exclusão social. Rev. Panam SaludPublica, v. 19, n. 6, p. 385-393, 2006.

23) RoncalliAG.Sheiham A, Tsakos G, Araújo-Souza GC, WattRG. Social Factors Associated with the Decline in Caries in Brazilian Children between 1996 and 2010. Caries Research, v. 50, n. 6, p. 551-559, 2016.

Artigo apresentado em outubro de 2019. Artigo aprovado em janeiro de 2020 Artigo publicado em maio de 2020 